\title{
THE THERMAL RESPONSE OF A PULSAR GLITCH: THE NONSPHERICALLY SYMMETRIC CASE
}

\author{
K. S. Cheng, ${ }^{1}$ Y. Li, ${ }^{1,2}$ and W. M. Suen ${ }^{3}$ \\ Received 1998 January 22; accepted 1998 March 25; published 1998 May 7
}

\begin{abstract}
We study the thermal evolution of a pulsar after a glitch in which the energy is released from a relatively compact region. A set of relativistic thermal transport and energy balance equations is used to study the thermal evolution, without making the assumption of spherical symmetry. We use an exact cooling model to solve this set of differential equations. Our results could differ significantly from those obtained under the assumption of spherical symmetry. Even for young pulsars with a hot core like the Vela pulsar, a detectable hot spot could be observed after a glitch if a large amount of energy is released in a small region close to the surface of the star. The results suggest that the intensity variation and the relative phases of hard X-ray emissions in different epochs may provide important information on the equation of state.
\end{abstract}

Subject headings: dense matter — stars: evolution — stars: interiors — stars: neutron — X-rays: stars

\section{INTRODUCTION}

The studies of the thermal evolution of pulsars are believed to provide vital information on the internal properties of the neutron stars (for a review, see, e.g., Tsuruta 1992). Theoretical cooling curves are often compared with the surface temperatures of pulsars with different ages. However, different pulsars clearly have different parameters, e.g., the rotation period $(P)$ and the surface magnetic field $(B)$, which may affect the cooling processes. Several proposed internal heating mechanisms, e.g., the frictional heating between the crustal superfluid and the crust (Alpar et al. 1984a; Shibazaki \& Lamb 1988), the crust cracking (Cheng et al. 1992), the chemical heating (Reisenegger 1995), etc., all depend on the pulsar parameters, i.e., $P$ and $B$. Furthermore, different pulsars have different internal properties, e.g., mass, equation of state, impurity content, etc., which strongly affect the cooling curve. Hence, ideally speaking, one would like to study the thermal evolution process of an individual pulsar. But realistically, the normal cooling process is extremely slow and cannot be followed observationally for an individual star.

The thermal evolution of a pulsar after a glitch is particularly interesting in that it fills in this gap. A large amount of energy could be suddenly released during the period jump (glitch). The energy will eventually be transported to the surface of the star and released in the form of a transient thermal X-ray emission. Several authors (Van Riper, Epstein, \& Miller 1991; Chong \& Cheng 1994; Hirano et al. 1997) have calculated the thermal evolution of pulsars after glitches, but they all assumed that the energy is generated in a spherical shell inside the star. They found that the glitches cannot produce very significant observed results for young pulsars like the Vela pulsar (Van Riper et al. 1991). However, at least part of the energy released in the glitch could be deposited in a compact region in the inner crust, by either superfluid unpinning in a small pocket where the vortex lines are piled up (see, e.g., Cheng et al. 1988 and Alpar \& Pines 1995) or crust cracking (see, e.g., Ruderman 1991 and Cheng et al. 1992). The transport of this amount of energy is clearly not spherical symmetric (although, to a good approximation, the background geometry is spherical). In this

\footnotetext{
${ }^{1}$ Department of Physics, University of Hong Kong, Hong Kong, China.

${ }^{2}$ High Energy Astrophysics Laboratory, IHEP, Beijing, China.

${ }^{3}$ Department of Physics, Campus Box 1105, Washington University, St. Louis, MO 63130.
}

Letter, we study the general case of the energy transport inside neutron stars after a glitch, without the spherical symmetry assumption. In $\S 2$, we derive the general relativistic nonspherical symmetric transport and energy balance equations. In $\S 3$, the necessary physics inputs and solution algorithms for solving this set of relativistic differential equations are described. In particular, three important factors, which strongly affect the thermal evolution of pulsars after a glitch, including the total energy released from a glitch, the depth at which the energy is released, and the area over which it is released, are discussed. Numerical results are presented in $\S 4$, with a brief discussion in $\S 5$.

\section{GENERAL RELATIVISTIC NONSPHERICAL SYMMETRIC THERMAL TRANSPORT AND ENERGY BALANCE EQUATIONS}

The Newtonian thermal transport and energy balance equations are given, respectively, by

$$
\boldsymbol{\nabla} \cdot \boldsymbol{F}=-C_{V} \frac{d T}{d t}=-n T \frac{d s}{d t}-Q_{v}
$$

and

$$
\nabla T=-\frac{\boldsymbol{F}}{K}
$$

where $\boldsymbol{F}$ is the energy flux, $C_{V}$ is the specific heat capacity, $T$ is the temperature, $n$ is the particle number density, $s$ is the specific entropy per particle, $Q_{\nu}$ is the neutrino emissivity per unit volume, and $K$ is the thermal conductivity.

To generalize the above equations to a relativistic situation, we make the following assumptions: (1) The neutron star is rotating slowly enough that the metric tensor describing the background spacetime can be written as (Tolman 1934) $d s^{2}=-e^{2 \Phi(r)} d t^{2}+e^{2 \Lambda(r)} d r^{2}+r^{2}\left(d \theta^{2}+\sin ^{2} \theta d \varphi^{2}\right)$, where $e^{\Lambda(r)}=\left(1-2 G m / r c^{2}\right)^{-1 / 2}$. (2) The diffusion limit is appropriate for the energy transport. (3) There are no other entropygenerating mechanisms besides diffusion, and second-order flux terms in the transport are negligible. (4) Fluid motion inside the star due to the thermal effects is negligible. These simplifying assumptions are clearly justified for the problem at hand. The energy transport is then governed by the following equations. The energy-momentum tensor can be written as $T^{\mu \nu}=$ 
$(\rho+P) U^{\mu} U^{\nu}+\rho g^{\mu \nu}+q^{\mu} U^{\nu}+U^{\mu} q^{\nu}$, where $U^{\mu}$ is the 4velocity of the fluid flow, $\rho$ is total energy density measured in the rest frame of the fluid, and $P$ is the pressure in the rest frame of the fluid. The heat flow is given by $q^{\mu}=-K\left(g^{\mu \nu}+\right.$ $\left.U^{\mu} U^{\nu}\right)\left(T,_{\nu}+T a_{\nu}\right)$, where $a_{\nu}=U^{\mu} U_{\nu ; \mu}$ is the 4-acceleration and $K=K(T)$ is the effective conductivity, e.g., $K \propto T^{3}(t, r, \theta, \varphi)$ for photon diffusion. Since we assume that there is no fluid motion, the 4-velocity of the fluid is $U^{\alpha}=\left(e^{-\Phi}, 0,0,0\right)$ and $a^{\alpha}=\left(0, e^{-2 \Lambda} \partial_{r} \Phi, 0,0\right)$. The 4-heat flow is given by $q^{\alpha}=$ $\left[0,-K e^{-2 \Lambda}\left(\partial_{r} T+T \partial_{r} \Phi\right),-\left(K / r^{2}\right) \partial_{\theta} T,-\left(K / r^{2} \sin ^{2}\right) \theta \partial_{\varphi} T\right]$. The energy-momentum tensor components related to the energy transport equation are the following: $T^{t t}=\rho U^{t} U^{t}=\rho e^{-2 \Phi}$, $T^{t r}=f^{r}=q^{r} U^{t}=-K e^{-\Phi-2 \Lambda}\left(\partial_{r} T+T \partial_{r} \Phi\right), \quad T^{t \theta}=f^{\theta}=$ $q^{\theta} U^{t}=-\left(K e^{-\Phi} / r^{2}\right) \partial_{\theta} T$, and $T^{t \varphi}=f^{\varphi}=q^{\varphi} U^{t}=$ $-\left(K e^{-\Phi} / r^{2} \sin ^{2} \theta\right) \partial_{\varphi} T$. To express the thermal transport equation in terms of local observables, we note that $T^{\hat{\alpha} \beta}=$ $\left(\partial \xi^{\hat{\alpha}} / \partial x^{\alpha}\right)\left(\partial \zeta^{\hat{\beta}} / \partial x^{\beta}\right) T^{\alpha \beta}$, where $d \xi^{\hat{\alpha}}=\left(e^{\Phi} d t, e^{\Lambda} d r, r d \theta, r \sin \right.$ $\theta d \varphi)$ are the unit-one forms and $d x^{\alpha}=(d t, d r, d \theta, d \varphi)$. Therefore, the relativistic thermal transport equation is given by

$$
\left(e^{\Phi} T\right)_{; i}=-\frac{e^{\Phi} F_{i}}{K}
$$

where the subscript "; $i$ " denotes the spatial covariant derivative on the constant time slice of the metric, with $i=r, \theta$, $\varphi, F_{i}=\left(T^{\hat{i}}, T^{\hat{i}}, T^{\hat{\varphi} \hat{\varphi}}\right)$ the locally measured energy flux in spherical coordinates $\left[T^{\hat{\hat{r}}}=e^{(\Lambda+\Phi)} f^{r}=-K e^{-\Phi-\Lambda} \partial_{r}\left(e^{\Phi} T\right), T^{\hat{\hat{\theta}}}=\right.$ $r e^{\Phi} f^{\theta}=-(K / r) \partial_{\theta} T$, and $T^{\hat{\hat{\varphi}}}=e^{\Phi} r \sin \theta f^{\varphi}=$ $\left.-(K / r \sin \theta) \partial_{\varphi} T\right]$. We can see that this equation (i) reduces to equation (2) when $\Phi$ and $\Lambda$ go to zero in the Newtonian limit and (ii) reduces to the equations of, e.g., Straumann (1984), with $\partial / \partial \theta=\partial / \partial \varphi=0$, in the spherical symmetric case.

The energy balance equation can be derived by the conservation of the energy-momentum tensor, namely, $0=T_{; \mu}^{t \mu}=$ $T_{; t}^{t t}+(2 / r) f^{r}+(\cos \theta / \sin \theta) f^{\theta}++\partial_{r} f^{r}+f^{r} \partial_{r} \Lambda+3 f^{r} \partial_{r} \Phi+$ $\partial_{\theta} f^{\theta}+\partial_{\varphi} f^{\varphi}$, where $T_{; t}^{t t}=(\partial \rho / \partial t) e^{-2 \Phi}=(\partial \rho / \partial \tau) e^{-\Phi}$. Here $\partial \rho / \partial \tau$ is the rate of change of energy density measured in the proper frame; it depends on the processes under consideration. For example, if only heat conduction is considered, one has $\partial \rho / \partial t=C_{V}(\partial T / \partial t)$, with $C_{V}$ being the heat capacity measured in the proper frame. We include neutrino emission, which leads to $\partial \rho / \partial t=C_{V}(\partial T / \partial t)+e^{\Phi} Q_{\nu}=\left(e^{-\Phi-\Lambda} / r^{2}\right) \partial_{r}\left(r^{2} f^{r} e^{\Lambda+3 \Phi}\right)$ $+\left(e^{2 \Phi} / \sin \theta\right) \partial_{\theta}\left(\sin \theta f^{\theta}\right)+e^{2 \Phi} \partial_{\varphi} f^{\varphi}$. Using the relations between $f^{\alpha}$ and $F^{\alpha}$, we obtain

$$
\begin{aligned}
& -\left(C_{V} \frac{d T}{d t}+e^{\Phi} Q_{\nu}\right)=\frac{e^{-(\Phi+\Lambda)}}{r^{2}} \frac{\partial}{\partial r}\left(r^{2} F^{\hat{r}} e^{2 \Phi}\right) \\
& +\frac{e^{\Phi}}{r \sin \theta} \frac{\partial}{\partial \theta}\left(F^{\hat{\theta}} \sin \theta\right)+\frac{e^{\Phi}}{r \sin \theta} \frac{\partial}{\partial \varphi} F^{\hat{\varphi}}
\end{aligned}
$$

It is very easy to see that the above equation again recovers the well-known spherical symmetric case, as well as the Newtonian limit.

\section{PHYSICS INPUTS AND NUMERICAL ALGORITHMS}

Physics inputs include a stellar model, thermal conductivity, heat capacity, neutrino emissivity, superfluidity, surface temperature treatment, and the position and amount of the energy released by a glitch. In our calculations, we divide a neutron star into two parts. The region from the neutron star center to the place where the mass density equals the nuclear density
$\rho_{N}=2.8 \times 10^{14} \mathrm{~g} \mathrm{~cm}^{-3}$ is defined as the core, while the region from $\rho_{N}$ to the boundary density $\rho_{b}=10^{9} \mathrm{~g} \mathrm{~cm}^{-3}$ is defined as the crust. The core is treated as isothermal with a timedependent temperature. In the crust region, the temperature after a glitch has both spatial and temporal variations; hence, an "exact" or "evolutionary" treatment is necessary (Nomoto \& Tsuruta 1987; Van Riper 1991). In our treatment, the energy flow and the spatial dependence of the temperature throughout the crust are followed, but the thermal effects on the stellar structures are neglected; i.e., the same hydrostatic stellar model is used throughout the evolution.

The stellar structure of a neutron star is determined by the equation of state (EOS). We consider three representative EOSs in this Letter, with the total mass of the neutron star in all three models taken to be $1.4 M_{\odot}$. The first EOS is the BPS model (Baym, Pethick, \& Sutherland 1971), which is often used as a soft EOS in neutron star cooling studies. The BPS model results in a high central density and little mass in the crust, and hence it has a small radius. The second EOS used is the PPS model (Pandharipande, Pines, \& Smith 1976), which is a stiff EOS with a low central density, a thick crust, and a large radius. The third EOS, the UT model (Wiringa \& Fiks 1988), is a representative intermediate stiff model. Its central density, crust thickness, and radius are between those of the BPS and PPS models.

In the crust region, we fitted the thermal conductivity data provided by Van Riper (1991), who followed the work of Itoh et al. (1984a, 1984b, 1984c, 1984d), together with the quantum corrections of Mitake, Ichimaru, \& Itoh (1984). The ions A and $\mathrm{Z}$, which are required in the conductivity formulae, are taken from Lattimer et al. (1985). It is important to note that the conductivity decreases as the temperature increases.

For a star with a core temperature $\sim 10^{8} \mathrm{~K}$, the important neutrino emission processes include electron bremsstrahlung, neutron-neutron, neutron-proton bremsstrahlung, and the modified Urca process. The rapid cooling processes, e.g., pion condensation (see, e.g., Maxwell 1979) or the direct Urca process, etc., will not be considered here.

The heat capacities in the crust region come from extreme relativistic degenerate electrons and from nonrelativistic neutrons and ions. The capacities of electrons and neutrons are given by Glen \& Sutherland (1980). The capacities of ions are given by Van Riper (1991). The capacities of the core are mainly due to the relativistic electrons, the superconducting protons, and the superfluid neutrons (Maxwell 1979). The transition temperature of normal-superfluid neutrons is given by Takatsuka \& Tamagaki (1971).

We use an empirical formula (Gudmundsson, Pethick, \& Epstein 1983) to relate the surface temperature $T_{s}$ and the boundary temperature $T_{b}$ at $\rho_{b}$. Eichler \& Cheng (1989) have shown that the thermal response time between the boundary and the surface is on the order of seconds, which is even less than our numerical time step (greater than $10 \mathrm{~s}$ ). The above relation is clearly valid in our computation.

The actual energy released from a glitch is not known. In fact, different models can give very different results. There are several such examples. First, the neutron star is known to consist of two components, superfluid and normal matter, which need not have the same angular velocity as the star spins down. Glitches may result from the sudden angular momentum transfer between these two components (Alpar et al. 1984a, 1984b). The energy released from a glitch in such a model is $\sim I_{c} \Delta \Omega_{c}\left(\Omega_{s}-\Omega_{c}\right)$, where $I_{c}$ is the moment of inertia of the crust and of those components strongly coupled to the crust, which 
is in general very close to the total stellar moment of inertia, $\Delta \Omega_{c}$ is the observed jump of the crustal angular velocity, and $\Omega_{c}-\Omega_{s}$ is the angular velocity lag between the crustal component and the superfluid. However, the angular velocity lag cannot be directly determined. The model-dependent estimation of this angular velocity lag ranges from $10^{-3}$ to $10 \mathrm{rad} \mathrm{s}^{-1}$ (Alpar et al. 1984a, 1984b; Epstein \& Baym 1988; Alpar, Cheng, \& Pines 1989). The glitch energy predicted by these models could be $10^{38}-10^{42}$ ergs. Van Riper et al. (1991) estimated that the energy released from the glitch is between $10^{41}$ and $10^{43} \mathrm{ergs}$ if the sudden angular momentum transfer takes place in the interstitial pinning region. Ruderman (1991) argued that the glitch may result from the crust cracking, and the energy released from the crust cracking is $\sim 10^{38} \theta_{-3}^{2}$ ergs over a small volume with a typical size of $10^{5} \mathrm{~cm}$. Here $\theta_{-3}$, in units of $10^{-3}$, is the maximum shear angle beyond which the crust will break. For a pure Coulomb lattice, $\theta$ is between 0.1 and 0.01 , but for an impurity-dominant lattice, $\theta$ can be as small as $10^{-5}$. It has been shown that even when $10^{43}$ ergs of glitch energy is released in a spherical shell inside a young pulsar with a hot interior, the temperature rise on the surface of the neutron star is undetectable (see, e.g., Van Riper et al. 1991 and Chong \& Cheng 1994). However, if a large amount of energy is indeed released in a small region close to the stellar surface, it may result in a detectable hot spot on the stellar surface. There are three crucial questions, namely, how much glitch energy is released in a small region, what is the depth at which this energy is released, and where is the area over which it is released. The answers to these questions are again very model dependent. A detailed discussion on the energy released from various glitch mechanisms will be presented elsewhere. In this Letter, we will assume the largest possible value of the glitch energy $\Delta E=10^{42}$ ergs, which is released at the depth of the crust $\sim 10^{12} \mathrm{~g} \mathrm{~cm}^{-3}$ over a typical size of $\sim 10^{5} \mathrm{~cm}$.

At time $t=0$, the heat is deposited in a small volume at $r=r_{g}$ and $\theta=0 ; r_{g}$ is the radius where the density is $\rho_{g}=$ $10^{12} \mathrm{~g} \mathrm{~cm}^{-3}$ (see Fig. 1). Without a loss of generality, we choose the hot spot to be centered at $\theta=0$, which gets rid of the $\varphi$ dependence in the transport problem. The transport equations in the crust region are solved by explicit finite-differencing, while the core is taken to be isothermal with a time-dependent temperature $T_{\text {core }}$ determined by the total heat inflow and the core heat capacity. In a typical run, the spatial resolution is taken to be $N_{r} \times N_{\theta}=100 \times 50$, with a variable time step determined by the stability requirements. The numerical convergence of the results has been checked carefully. The temperature is cell-centered, while the flux is centered on the cell surface. The inner and outer boundary conditions for the flux are given explicitly by

$$
\begin{gathered}
F^{r}(1, j)=-K e^{-\Phi-\Lambda} \frac{e^{\Phi(1, j)} T(1, j)-e^{\Phi(\mathrm{core})} T_{\text {core }}}{\Delta r}, \\
1 \leq j \leq N_{\theta}
\end{gathered}
$$

at the inner boundary and

$$
F^{r}\left(N_{r}+1, j\right)=\sigma T_{s}^{4}\left(N_{r}, j\right) \frac{r_{s}^{2}}{r_{\mathrm{ob}}^{2}}, 1 \leq j \leq N_{\theta},
$$

at the outer boundary; $r_{\mathrm{ob}}$ and $r_{s}$ are, respectively, the radii of the outer boundary of the crust and the star; $\sigma$ is the StefanBoltzmann constant; $T_{s}\left(N_{r}, j\right)$ is the surface temperature of the

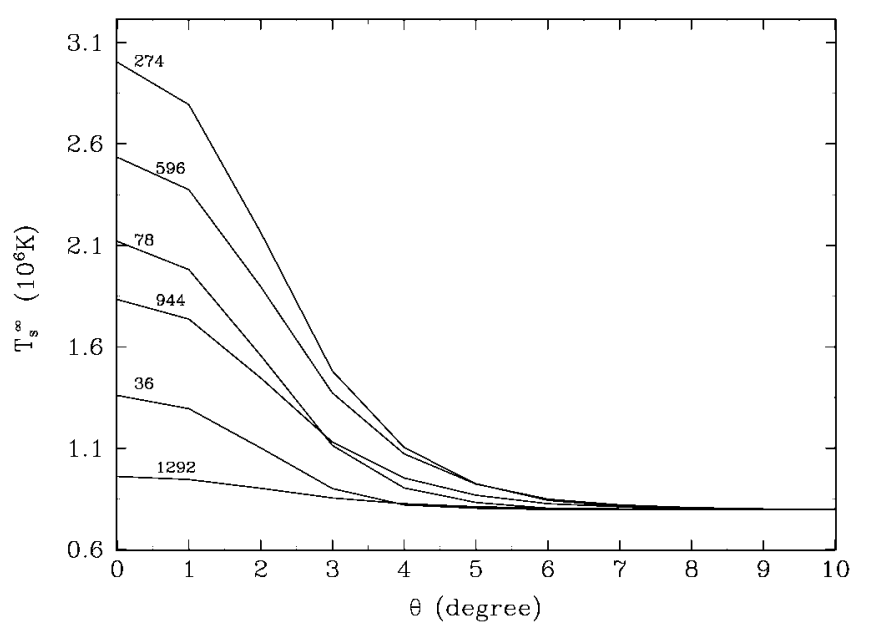

FIG. 1.-The thermal profiles of the hot spot on the surface of the neutron star of the UT EOS labeled by the time in days after a heat input of $\Delta E=$ $10^{42}$ ergs induced by a glitch released at $3 \times 10^{11} \mathrm{~g} \mathrm{~cm}^{-3} \leq \rho \leq 3 \times 10^{12} \mathrm{~g}$ $\mathrm{cm}^{-3}$ in a nonspherical symmetric case as a function of polar angle. The core temperature is $T_{c}=10^{8} \mathrm{~K}$.

$j$ th angular cell at $r_{\mathrm{ob}}$. The initial temperature distribution within the crust is that of the equilibrium state of the same star with the initial core temperature. The temperature evolution is given by standard finite-differencing of equation (4). With a secondorder scheme, no extra boundary condition is needed for equation (4) since the temperature is cell-centered.

\section{RESULTS}

We believe the energy is released at $3 \times 10^{11} \mathrm{~g} \mathrm{~cm}^{-3} \leq$ $\rho \leq 3 \times 10^{12} \mathrm{~g} \mathrm{~cm}^{-3}$, with a solid angle of $2^{\circ} \times 2^{\circ}$ centered at $\theta=0^{\circ}$. The initial temperature of the core is taken to be $T_{c}=10^{8} \mathrm{~K}$, and the released energy $\Delta E=10^{42} \mathrm{ergs}$, which is about the energy released by the Vela pulsar after a giant glitch. Figure 1 shows the surface temperature versus the polar angle at different times. The hot spot is gradually spreading away from $\theta=0^{\circ}$, and it takes about 275 days for the surface temperature to reach the maximum for a UT star. The temperature at maximum is about 5 times higher than the background temperature. However, the hottest region is concentrated in a solid angle of $2^{\circ} \times 2^{\circ}$ centered at $\theta=0^{\circ}$.

Figure 2 shows the evolution of the surface luminosity as function of time for three different EOSs, i.e., PPS, UT, and BPS. The peak luminosity is higher, the time needed to reach the peak is shorter, and the relaxation time is also shorter for the softer EOS in comparison with the stiff one. This is true for the following reason: the soft EOS has a much thinner crust. We can see that there are substantial differences among these EOSs.

In comparing the luminosity evolution between the spherical symmetric case and the nonspherical symmetric case (cf. Fig. 1 of Chong \& Cheng 1994, hereafter CC94, with our Fig. 2), we find that there are three major differences between these two cases: (1) The surface luminosity of the spherical symmetric case reaches the peak almost 5 times faster than that of the nonspherical symmetric case. This results from the fact that the nonspherical symmetric case has a much higher temperature that decreases the conductivity considerably. (2) The peak of the total luminosity in the spherical case is lower than that of the nonspherical symmetric case. This is so because in the nonspherical case, more energy comes out through the surface 


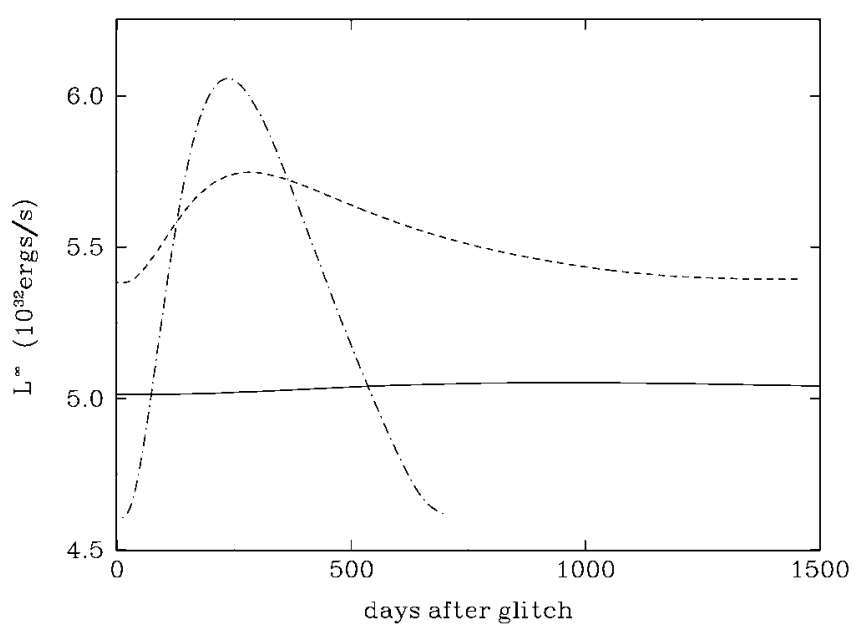

FIG. 2.-The evolution curves of the total luminosity for PPS, UT, and BPS stars denoted by solid, dashed, and dot-dashed lines, respectively.

instead of heating the core. (3) Most importantly, although the changes of the total luminosity for the spherical case and the nonspherical case are very small, the surface temperature of the nonspherical symmetric case at a $2^{\circ} \times 2^{\circ}$ cap area changes drastically (cf. our Fig. 1 with Fig. $3 c$ of CC94), while the surface temperature changes very little in the spherical symmetric case. In other words, a hot spot should show up on the surface of a neutron star 200-300 days after the glitch, and it should last for about a few hundred days if the EOS is soft or intermediately stiff.

In calculating the evolution of the total luminosity for different core temperatures, we find that the surface luminosity of the cooler model reaches its maximum earlier. This results from the fact that the conductivity is higher for lower temperature. The relative increase in the luminosity of the cooler star is higher than that of the hotter star. The net increase of the luminosity of the cooler star is a little higher than that of the hotter star. It is true for the following reason: the energy of the glitch spreads to the stellar surface of the cooler star faster than that of the hotter star. However, the total energy emitted during the thermal afterglow period is about the same in these two cases. We have also calculated the thermal evolution inside the neutron star at $\theta=0^{\circ}$ (cf. Fig. 1 of Cheng \&
Li 1998). In comparing this with the spherical symmetric cases (see, e.g., Fig. 2 of CC94), we find that the temperature of the heat pulse is much higher in this case because the energy is released in a much smaller volume. The heat pulse is propagating outward as well as inward, with a speed slower than that of Figure 2 of CC94 (where the core temperature is chosen to be $10^{6} \mathrm{~K}$, and hence the conductivity is much larger).

\section{DISCUSSION}

Based on a set of general relativistic thermal transport and energy balance equations, we studied the thermal evolution of a neutron star after a glitch. There are large uncertainties in some factors that strongly affect the thermal response of a pulsar after a glitch, i.e., the total energy released from a glitch, the depth at which the energy is released, and the area over which it is released.

For illustrative purposes, we have assumed that there is $10^{42}$ ergs of glitch energy released in a compact region with a typical size $10^{5} \mathrm{~cm}$ at density $\sim 10^{12} \mathrm{~g} \mathrm{~cm}^{-3}$; then a hot spot can appear on the stellar surface. For a UT star with an interior temperature of $\sim 10^{8} \mathrm{~K}$, although the surface luminosity increases by only $\sim 10 \%$, the radiation is emitted from a small area with a temperature higher than the background temperature by a factor of $\sim 5$. This results in a periodic hard $\mathrm{X}$-ray pulse emission that should stand out clearly from the soft X-ray background. A soft EOS greatly enhances this effect, and a stiff EOS reduces it: the thermal response to a glitch can provide important constraints on the EOS.

The time it takes to reach the peak luminosity is long, typically $\sim 1 \mathrm{yr}$ for a UT star; this may make it difficult to relate the hard X-ray pulse to the glitch generating it. However, since the energy released by each glitch should be at a different place on the star, comparing the relative pulse-phase difference with the intensity variation of the hard X-rays observed in different epochs can provide evidence for this phenomenon. Together with a detailed spectral analysis, the EOS could be deduced. A detailed report on this subject and a comparison with observed data will be presented elsewhere.

We thank M. A. Alpar, Q. Lu, A. Reisengger, M. Ruderman, and N. Shibazaki for interesting discussions and comments. K. S. C. and Y. L. are supported by an RGC grant from the Hong Kong Government.

\section{REFERENCES}

Alpar, M. A., Anderson, P. W., Pines, D., \& Shaham, J. 1984a, ApJ, 276, 325 1984b, ApJ, 278, 791

Alpar, M. A., Cheng, K. S., \& Pines, D. 1989, ApJ, 346, 823

Alpar, M. A., \& Pines, R. 1995, in NATO/ASI Ser. C, The Lives of the Neutron

Stars, ed. M. A. Alpar, Ü. Kiziloğlu, \& J. van Paradijs (Dordrecht: Kluwer), 185

Baym, G., Pethick, C. J., \& Sutherland, P. G. 1971, ApJ, 170, 299

Cheng, K. S., Chau, W. Y., Zhang, J. L., \& Chau, H. F. 1992, ApJ, 396, 135

Cheng, K. S., \& Li, Y. 1998, in ASP Conf. Ser. 138, 1997 Pacific Rim Con-

ference on Stellar Astrophysics, ed. K. L. Chan, K. S. Cheng, \& H. P. Singh (San Francisco: ASP), in press

Cheng, K. S., Pines, D., Alpar, M. A., \& Shaham, J. 1988, ApJ, 330, 835

Chong, N., \& Cheng, K. S. 1994, ApJ, 425, 210 (CC94)

Eichler, D., \& Cheng, A. F. 1989, ApJ, 336, 360

Epstein, R. I., \& Baym, G. 1988, ApJ, 328, 680

Glen, G., \& Sutherland, P. G. 1980, ApJ, 239, 671

Gudmundsson, E. H., Pethick, C. J., \& Epstein, R. I. 1983, ApJ, 272, 286

Hirano, S., Shibazaki, N., Umeda, H., \& Nomoto, K. 1997, ApJ, 491, 286

Itoh, N., Kohyama, Y., Matsumoto, N., \& Seki, M. 1984a, ApJ, 279, 413 1984b, ApJ, 280, 787

$1984 \mathrm{c}$, ApJ, 285, 758
Itoh, N., Matsumoto, N., Seki, M., \& Kohyama, Y. 1984d, ApJ, 273, 774

Lattimer, J. M., Pethick, C. J., Ravenhall, D. G., \& Lamb, D. Q. 1985, Nucl. Phys. A, 432, 646

Maxwell, O. V. 1979, ApJ, 231, 201

Mitake, S., Ichimaru, S., \& Itoh, N. 1984, ApJ, 277, 375

Nomoto, K., \& Tsuruta, S. 1987, ApJ, 312, 711

Pandharipande, V. R., Pines, D., \& Smith, R. A. 1976, ApJ, 208, 550

Reisenegger, A. 1995, ApJ, 442, 749

Ruderman, M. A. 1991, ApJ, 366, 261

Shibazaki, N., \& Lamb, F. K. 1988, ApJ, 346, 808

Straumann, N. 1984, General Relativity and Relativistic Astrophysics (Berlin: Springer)

Takatsuka, T., \& Tamagaki, R. 1971, Prog. Theor. Phys., 46, 114

Tolman, R. C. 1934, Relativity, Thermodynamics, and Cosmology (Oxford: Clarendon)

Tsuruta, S. 1992, in Conf. Proc., The Structure and Evolution of Neutron Stars, eds. D. Pines, R. Tamagaki, \& S. Tsuruta (Kyoto: Addison-Wesley), 317

Van Riper, K. A. 1991, ApJ, 75, 449

Van Riper, K. A., Epstein, R. I., \& Miller, G. S. 1991, ApJ, 381, L47

Wiringa, R. B., \& Fiks, V. 1988, Phys. Rev. C, 38, 1010 